\title{
Asiaticoside ameliorates $\beta$-amyloid-induced learning and memory deficits in rats by inhibiting mitochondrial apoptosis and reducing inflammatory factors
}

\author{
ZHUO ZHANG ${ }^{1}, \mathrm{XIAOBIN} \mathrm{LI}^{1}, \mathrm{DUO} \mathrm{LI}^{2}, \mathrm{MAOLUO}^{3}, \mathrm{YONGJIE} \mathrm{LI}^{3}, \mathrm{LI} \mathrm{SONG}^{4}$ and XIAN JIANG ${ }^{4}$ \\ ${ }^{1}$ Department of Pharmacology, Luzhou Medical College; ${ }^{2}$ Department of Respiratory Internal Medicine, \\ The First Affiliated Hospital of Luzhou Medical College; ${ }^{3}$ Department of Research Centre of Medicine and Functional Foods, \\ Luzhou Medical College; ${ }^{4}$ Department of Anesthesiology, The First Affiliated Hospital of Luzhou Medical College, \\ Luzhou, Sichuan 646000, P.R. China
}

Received August 15,2015; Accepted August 23, 2016

DOI: $10.3892 /$ etm.2016.4004

\begin{abstract}
The present study aimed to investigate the effects of asiaticoside (AS) on the pathology and associated mechanisms of $\beta$-amyloid (A $\beta$ )-induced Alzheimer's disease (AD) in rats. An AD rat model was established by lateral intracerebroventricular injection of $A \beta$ 1-42 oligomers. Learning and memory function were evaluated by Morris water maze (MWM) test. In addition, hematoxylin and eosin (H\&E) staining, transmission electron microscopy (TEM), immunohistochemistry, ELISA and western blot analysis were performed to evaluate the disease pathogenesis. The results indicated that AS exerted protective effects in rats treated with $A \beta$ oligomers, in a dose-dependent manner, as evidenced by the improved learning and memory function in the MWM test. In addition, H\&E staining of hippocampal tissue showed that the histological structure was damaged in the model group, which was restored by AS treatment. A $\beta$ deposition was dramatically increased in the model group, and the pathological changes were reversed by AS treatment. TEM revealed that the subcellular structure was injured by $\mathrm{A} \beta$ oligomers, however, the structure was ameliorated by AS treatment. Furthermore, AS was found to reduce the elevated levels of pro-inflammatory cytokines, interleukin- 6 and tumor necrosis factor- $\alpha$, in the brains of $A \beta$-treated rats. In addition, AS treatment resulted in a significant decrease in the expression of caspases-3, whereas the expression of B-cell lymphoma-2 was significantly increased, in these $\mathrm{A} \beta$-treated rats. According to the findings of the observed study, AS has a marked protective effect on $\mathrm{A} \beta$-induced AD pathology, and the underlying mechanism
\end{abstract}

Correspondence to: Professor Xian Jiang, Department of Anesthesiology, The First Affiliated Hospital of Luzhou Medical College, 319 Zhongshan Road, Luzhou, Sichuan 646000, P.R. China E-mail: jxian777@163.com

Key words: asiaticoside, $\beta$-amyloid, caspase-3, B-cell lymphoma-2, anti-inflammatory may be associated with the alleviation of the mitochondrial injuries, the anti-inflammatory activities, and the influence on the expression levels of apoptosis-associated proteins.

\section{Introduction}

Asiaticoside (AS), a triterpenoid derivative isolated from Centella asiatica, has versatile biological effects, including antioxidant and anti-inflammatory activities, as well as protective effects against glutamate- or $\beta$-amyloid (A $\beta)$-induced neurotoxicity (1). In our previous study, AS has been shown to protect against apoptosis induced by $\mathrm{A} \beta 25-35$ in PC12 cells, and increase the cell viability and the expression level of B-cell lymphoma 2 (Bcl-2), in a dose-dependent manner (2). Furthermore, AS has marked hepatoprotective effects on liver injury induced by lipopolysaccharide/D-galactosamine $\mathrm{N}$, inhibiting the expression of tumor necrosis factor- $\alpha$ (TNF- $\alpha$ ) and mitogen-activated protein kinases (3).

Alzheimer's disease (AD) is a disorder of the neural system that is characterized by the irreversible decline of cognitive function (4). The mechanism of AD development is complex, and $A \beta$ peptide may serve a central role in the disease pathogenesis. AD develops as a result of the overproduction and aggregation of $A \beta$ in the brain. $A \beta$ peptides can cause mitochondrial dysfunction and caspase activation, leading to apoptosis in neuronal cells (5). Mitochondria are metabolically active in living cells, and these organelles respond to various pathophysiological cues to provide cellular energy and meet metabolic demand (6-8). When the mitochondria are severely impaired, apoptosis and/or necrosis may occur, resulting in cell death (9-11). Concerning the effects of AS on cell viability and the role of mitochondrion-associated apoptosis in AD pathogenesis, it is of great importance to examine the effects of AS on the disease pathogenesis and the underlying mechanisms.

In the present study, the effects of AS on A $\beta$-induced AD pathology in rats were investigated, and the possible underlying mechanisms were also discussed. The present findings suggest the potential application of AS on AD in clinical practice in the future. 


\section{Materials and methods}

Animals and grouping. A total of 60 adult male Sprague-Dawley rats (age, 12 weeks; weight, 220-260 g) were obtained from the Laboratory Animal Centre of South West Medical University (Chongqing, China). All the experimental procedures were performed according to the ethical guidelines of Luzhou Medical College (Luzhou, China) for the care and use of laboratory animals. This study was approved by the Ethics Committee board of Luzhou Medical College. Rats were housed in an air-conditioned room at $25^{\circ} \mathrm{C}$, with $55 \%$ humidity and a 12-h light/dark cycle, and fed with standard diet. They were acclimated for 5 days prior to the experiments.

The rats were randomly divided into the following groups ( $n=10$ in each group): i) Control group, which was treated with only saline; ii) model group, in which rats were subjected to lateral intracerebroventricular infusion with $A \beta$ 1-42 oligomers; iii) sham group, in which rats were infused with normal saline instead of $A \beta$ 1-42 oligomers; and iv) three treatment groups, in which the rats were infused with $A \beta$ 1-42 oligomers for 14 days, followed by the drug treatment with 5,15 or $45 \mathrm{mg} / \mathrm{kg}$ AS.

A $\beta$ 1-42 oligomer preparation and animal modeling. Synthetic A $\beta$ 1-42 peptides (Sigma-Aldrich, St. Louis, MO, USA) were dissolved in phosphate buffer solution at a final concentration of $0.45 \mathrm{mg} / \mathrm{ml}$ (or $100 \mu \mathrm{mol} / \mathrm{l}$ ), and then stored at $-80^{\circ} \mathrm{C}$. The solution containing oligomers was incubated at $37^{\circ} \mathrm{C}$ for 7 days (12-14). Purity control and structure proof were achieved with amino acid analysis and electrospray mass spectrometry (Finnigan Mat TSQ 7000; Thermo Fisher Scientific, Inc., Waltham, MA, USA) as previously described (15).

To establish the AD model, rats were initially anesthetized with intraperitoneal injection of 3\% pentobarbital sodium (30 mg/kg body weight; Sigma-Aldrich), and then mounted on a stereotaxic apparatus (ZH-B; Shanghai Zhenghua Medical Equipment Co., Ltd., Shanghai, China). A $1.5-\mathrm{cm}$ incision was made on the scalp, and the anterior fontanel was exposed. The coordinates were as follows (from the bregma): Anterior-posterior, $3.5 \mathrm{~mm}$; medial-lateral, $2.0 \mathrm{~mm}$; and dorsal-ventral, $2.7 \mathrm{~mm}$ (16). Subsequently, $10 \mu \mathrm{l} \mathrm{A} \beta$ 1-42 $(1 \mu \mathrm{mol})$ was bilaterally infused with a microsyringe over a period of $10 \mathrm{~min}$, and the microsyringe was kept for another 5 min before withdrawal. In the sham group, $1 \mu 1$ normal saline was infused rather than $A \beta 1-42$, following the same procedure.

Drug administration. Asiaticoside (AS; $\mathrm{C}_{48} \mathrm{H}_{78} \mathrm{O}_{19} ;$ molecular weight, 959.12; purity, >98\%) was purchased from Guangxi Changzhou Natural Product Development Co., Ltd. (Nanning, China), and determined by high-performance liquid chromatography as previously described (1/Qi). The drug administration was performed after the AD model was established using $\mathrm{A} \beta$ oligomer treatment. AS was administered orally at 5,15 or $45 \mathrm{mg} / \mathrm{kg}$ body weight per day for 7 days.

Morris water maze (MWM) test. The learning and memory function of the rats were evaluated by the MWM test 7 days after surgery, according to a previously described method (17). The swimming pool (180 cm in diameter) was maintained at $22-24^{\circ} \mathrm{C}$, and divided into four quadrants with equal size. A hidden platform was placed in the center of one quadrant. Each of the cardinal points of these four quadrants was randomly selected as the start location. On the pre-test day, rats were allowed to swim freely for $120 \mathrm{sec}$. During the test (days 1-4), each rat was subjected to 8 trials each day. The trial began when a rat was placed in the pool, and ended when the rat found the platform. The escape latency was recorded. If a rat failed to find the platform within $120 \mathrm{sec}$, the trial was terminated and the escape latency was recorded as $120 \mathrm{sec}$, and the rat was guided to the platform $(18,19)$. On day 5 , the swimming path was recorded by a video recording system, and the digital images were analyzed by the water maze software (Taimeng Technology Co., Ltd., Chengdu, China). In addition, the probe test was performed, in which the platform was removed, and the rat was allowed to swim freely in the pool for $120 \mathrm{sec}$. The swimming time in the target quadrant was recorded.

Hematoxylin and eosin $(H \& E)$ staining. After the behavioral test on day 12 after surgery, the rats were deeply anesthetized with intraperitoneal injection of $3 \%$ pentobarbital sodium (30 mg/kg body weight). The hippocampi were removed and fixed with $10 \%$ neutral-buffered formalin for 3 days, and then embedded in paraffin. Next, the samples were cut into $5-\mathrm{mm}$ sections on a rotary microtome (Leitz 1512; Leica Microsystems, Wetzlar, Germany). Sections were then subjected to $\mathrm{H} \& \mathrm{E}$ staining according to the standard protocol, and were observed with light microscopy.

Immunohistochemistry. On day 12 after surgey, hippocampi were removed from the rats and fixed with $10 \%$ neutral-buffered formalin for 3 days, and then embedded in paraffin. The tissues were sliced at $10 \mu \mathrm{m}$ and mounted on a slide glass. The slides were dewaxed, heated to $100^{\circ} \mathrm{C}$ for $30 \mathrm{sec}$ in $0.01 \mathrm{M}$ citric acid buffer ( $\mathrm{pH}$ 6.0), and then cooled at room temperature. After blocking with 5\% goat serum, the sections were incubated with rabbit anti-rat anti-A $\beta$ 1-42 polyclonal antibody (1:200; 20141020; Beijing Biosynthesis Biotechnology Co., Ltd., Beijing, China), at $4^{\circ} \mathrm{C}$ overnight. Subsequent to washing with phosphate-buffered saline, the section was stained with biotin-conjugated goat anti-rabbit polyclonal $\operatorname{IgG}(1: 100$; 20140321; Beijing Biosynthesis Biotechnology Co., Ltd.) for $30 \mathrm{~min}$, and then examined under a fluorescent microscope.

Western blot analysis. Frozen hippocampal samples were homogenated with triple detergent lysis buffer, containing $50 \mathrm{mM}$ Tris- $\mathrm{HCl}$ (pH 7.5), $150 \mathrm{mM} \mathrm{NaCl}, 0.1 \%$ sodium dodecyl sulfate (SDS), $1 \%$ Nonidet P-40, 0.5\% sodium deoxycholate, $100 \mathrm{mg} / \mathrm{ml}$ phenylmethylsulfonyl fluoride and $1 \mathrm{mg} / \mathrm{ml}$ aprotinin. Protein concentration was determined by the BCA protein assay kit (Pierce; Thermo Fisher Scientific, Inc.). Protein samples were separated by $12 \%$ SDS-polyacrylamide gel electrophoresis, and then electronically transferred onto a nitrocellulose membrane. The membrane was blocked with $5 \%(\mathrm{w} / \mathrm{v})$ fat-free milk in Tris-buffered saline containing $0.05 \%$ Tween-20, followed by incubation with rabbit anti-Bcl-2 (1:5,000; ab32314; Abcam, Cambridge, MA, UK), anti-caspase-3 (1:5,000; ab13847; Abcam), or anti- $\beta$-actin (ab8227; Abcam) polyclonal antibodies. at $4^{\circ} \mathrm{C}$ overnight. Subsequently, the membrane was incubated with horseradish peroxidase-conjugated goat anti-rabbit secondary 
$\mathbf{A}$

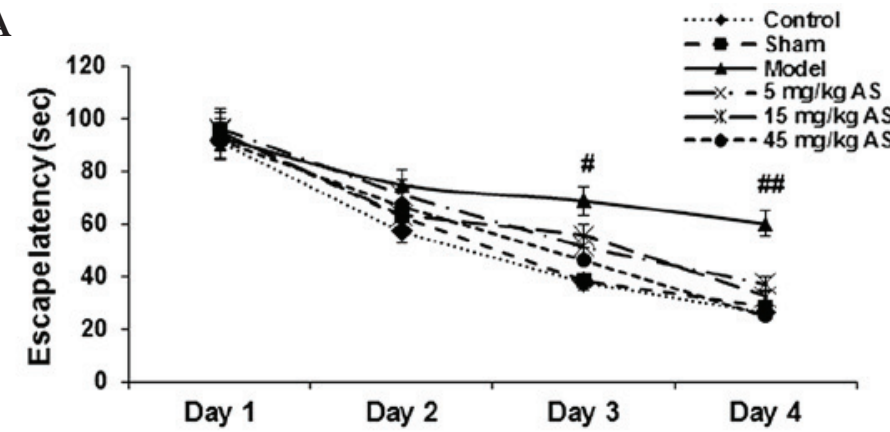

B

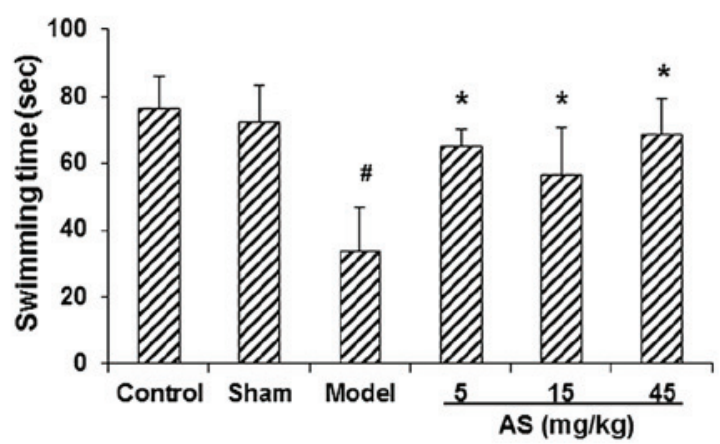

C

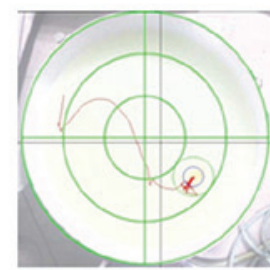

Control

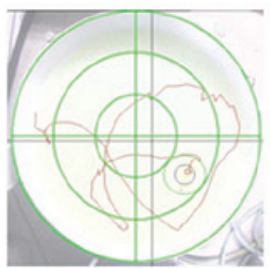

Sham

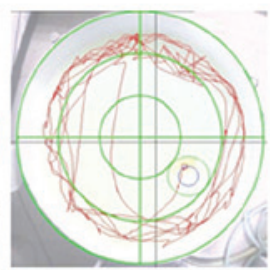

Model

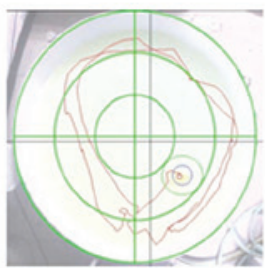

$5 \mathrm{mg} / \mathrm{kgAS}$

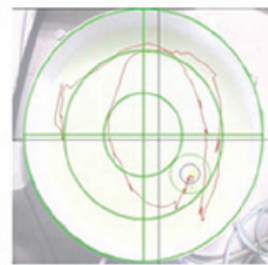

$15 \mathrm{mg} / \mathrm{kg}$ AS

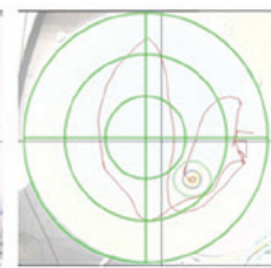

$45 \mathrm{mg} / \mathrm{kg} \mathrm{AS}$

Figure 1. AS treatment ameliorated the learning and memory deficit in A $\beta$-treated rats. Morris water maze test was performed for 5 days to assess the learning and memory function of A $\beta$-treated rats. The (A) escape latency, (B) swimming time in the target quadrant, and (C) navigation path in the various study groups are shown. ${ }^{\#} \mathrm{P}<0.05$ and $^{\# \#} \mathrm{P}<0.01$, vs. control group. AS, asiaticoside; $\mathrm{A} \beta, \beta$-amyloid

antibody (1:3,000; Cat. No., bs0295g-HRP; Beijing Biosynthesis Biotechnology Co., Ltd.). Protein bands were visualized with an enhanced chemiluminescence system (GelDocXR+; V140130; Bio-Rad Laboratories, Inc., Hercules, CA, USA). Protein bands on the images and the relative protein expression were analyzed using Quantity One software (Version 4.62).

Transmission electron microscopy (TEM). The subcellular structure of the brain was detected with TEM. Briefly, rats were anesthetized and rapidly perfused with $100 \mathrm{ml}$ solution containing $0.85 \mathrm{~g}$ sodium chloride, $0.025 \mathrm{~g}$ potassium chloride and $0.02 \mathrm{~g}$ sodium bicarbonate. Next, they were perfused with $300 \mathrm{ml}$ fixing solution containing $0.5 \%$ glutaraldehyde and $4 \%$ paraformaldehyde. The entire brain was removed, fixed overnight with $0.5 \%$ glutaraldehyde and $4 \%$ paraformaldehyde, then embedded in paraffin. The sample was stained with uranium-lead (20070112; Lihua Kaishun, Beijing, China)., and observed using a transmission electron microscope.

Microdialysis. Microdialysis probes (CMA 20;0.5-mm diameter, $10-\mathrm{mm}$ length, $100 \mathrm{kDa}$ cutoff polyethersulfone membrane; CMA Microdialysis AB, Solna, Sweden) were injected into the brains of the rats on day 12 after surgery at $0.6 \mu \mathrm{l} / \mathrm{min}$, with $0.9 \%(\mathrm{w} / \mathrm{v})$ saline containing $40 \mathrm{mg} / \mathrm{ml}$ dextran (Meda AB, Solna, Sweden), as previously described $(20,21)$. Following a 30-min equilibration period, microdialysate was collected on ice and stored at $-70^{\circ} \mathrm{C}$. Next, the concentrations of interleukin-6 (IL-6) and TNF- $\alpha$ in the brain microdialysate were determined.

Enzyme-linked immunosorbent assay (ELISA). The concentrations of IL- 6 and TNF- $\alpha$ in brain microdialysate were quantified using an ELISA kit ( 20140127; R\&D Systems, Minneapolis, MN, USA), according to the manufacturer's instructions. IL- 6 and TNF- $\alpha$ levels were obtained according to the standard curve. All experiments were performed in triplicate.

Statistical analysis. Data are expressed as the mean \pm standard deviation. Statistical analysis was performed with SPSS version 17.0 software (SPSS, Inc., Chicago, IL, USA). Comparisons for multiple groups were performed using one-way analysis of variance, and comparisons between groups were performed using Student-Newman-Keuls method. P $<0.05$ was considered to indicate statistically significant differences among the groups.

\section{Results}

AS ameliorates the learning and memory deficit in A $\beta$-treated rats. To investigate the effects of AS on the learning and memory function of $A \beta$-treated rats, the MWM test was performed. The results demonstrated that the escape latencies of rats in the various groups did not reveal statistically significant differences on day 1 (Fig. 1A). However, on days 2-4, the escape latency of the model group was significantly greater compared with that in the control and sham groups $(\mathrm{P}<0.01$; Fig. $1 \mathrm{~A})$. Following treatment with 15 and $45 \mathrm{mg} / \mathrm{kg}$ body weight AS, the escape latencies of the $\mathrm{A} \beta$-treated rats were significantly reduced $(\mathrm{P}<0.01$; Fig. $1 \mathrm{~A})$. Furthermore, according to the results of the swimming time in the target quadrant (Fig. 1B) and the navigation path recording (Fig. 1C), the cognitive capabilities were evidently declined in the model group compared with the control and sham groups. The cognitive capability was restored by the treatment with 5 , 15 or $45 \mathrm{mg} / \mathrm{kg}$ body weight AS $(\mathrm{P}<0.01)$. These results suggest that AS may ameliorate the learning and memory deficit in rats with $\mathrm{A} \beta$-induced $\mathrm{AD}$, in a dose-dependent manner.

AS protects hippocampi against the impairment caused by $A \beta$ oligomers. To investigate the effects of $A \beta$ oligomers and 


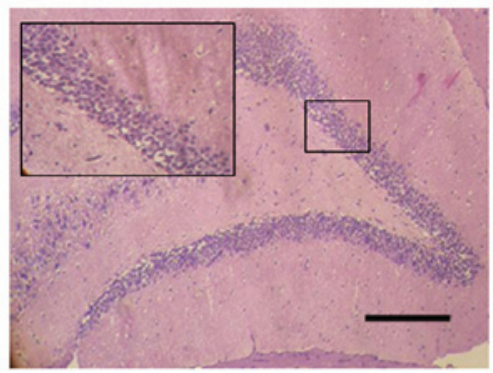

Control

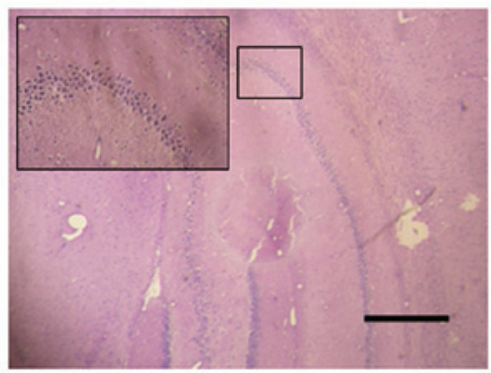

$5 \mathrm{mg} / \mathrm{kg}$ AS

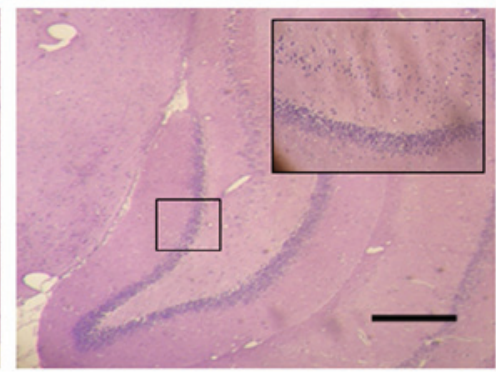

Sham

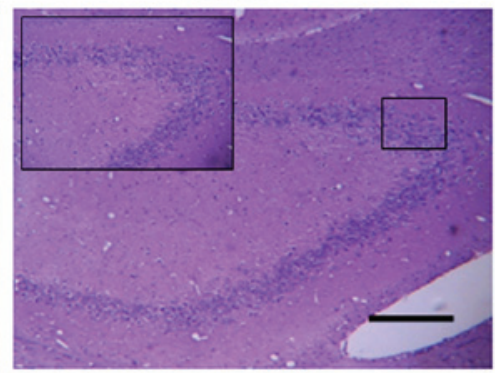

$15 \mathrm{mg} / \mathrm{kg}$ AS

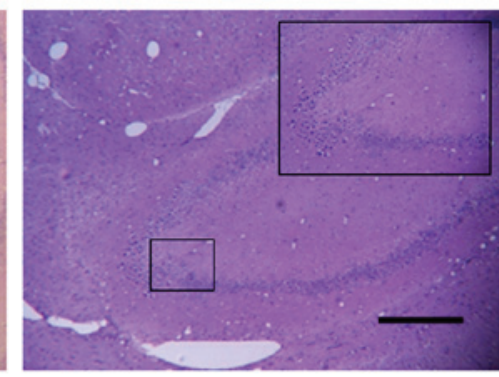

Model

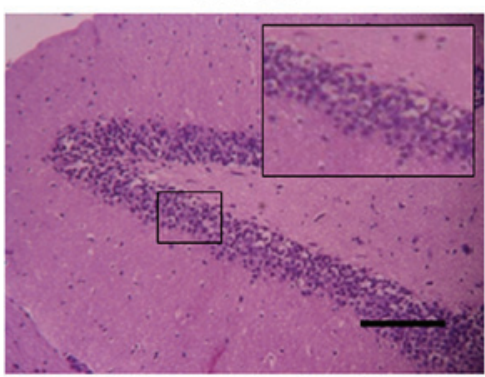

$45 \mathrm{mg} / \mathrm{kg}$ AS

Figure 2. AS treatment restored the impaired histological structure in rats treated with $\beta$-amyloid. Hematoxylin and eosin staining was performed to detect the histological structure of the hippocampus of rats in the control, sham, model and AS treatment $(5,15 \mathrm{or} 45 \mathrm{mg} / \mathrm{kg}$ body weight) groups. Scale bar, $10 \mu \mathrm{m}$. AS, asiaticoside.

AS on hippocampi in rats, H\&E staining was performed. The results indicated that, in the control and sham groups, complete hippocampal neurons and cells in surrounding tissues were observed, with integrated cell membrane, uniform-colored cytoplasm and round nuclei. However, in the model group, the structure of hippocampal neurons was incomplete, the connection with cells in the surrounding tissues was weak, and edema and bubbles were observed, indicating cell death. By contrast, treatment with 5,15 and $45 \mathrm{mg} / \mathrm{kg}$ body weight AS significantly ameliorated the impairments in the hippocampal region caused by $\mathrm{A} \beta$ oligomers (Fig. 2), in a dose-dependent manner. These results suggest that AS treatment protected the hippocampi from $\mathrm{A} \beta$-induced impairment.

AS decreases $A \beta$ deposits in the hippocampus of rats treated with $A \beta$ oligomers. To further investigate the effects of AS treatment on $A \beta$-induced pathology in rats, immunohistochemistry was performed. The results demonstrated that, compared with the control and sham groups, the $A \beta$ deposits were significantly increased in the model group (Fig. 3). However, when treated with $\mathrm{AS}$, the numbers of $\mathrm{A} \beta$ deposits in the hippocampus of these model rats was significantly deceased (Fig. 3). These results suggest that AS reversed the pathological changes in the hippocampal region of $A \beta$-treated rats.

AS ameliorates impaired subcellular structure in rats treated with $A \beta$ oligomers. To investigate the effects of $A \beta$ oligomers and AS treatment on the subcellular structure in rat brains, TEM was performed. The TEM results indicated that the nuclear structure in hippocampal neurons was intact in the control and sham groups, with a clear double membrane, complete outer and inner membranes of mitochondria and intact cristae observed. However, in the model group, the hippocampal neurons were evidently damaged, exhibiting abnormal nuclear membrane and swelling mitochondria with vague cristae (Fig. 4). The treatment with AS significantly ameliorated the pathological changes in the subcellular structure of hippocampal neurons that was induced by $A \beta$ oligomers. The nuclear membrane was clear and intact, while the outer and inner membranes of mitochondria were complete, with normal cristae. These results suggest that AS treatment ameliorated the impairments in the subcellular structure in the rats treated with $\mathrm{A} \beta$ oligomers.

$A S$ reduces $I L-6$ and TNF- $\alpha$ levels in the brains of $A \beta$-treated rats. To investigate the inflammatory status in the rats treated with $A \beta$ oligomers, the levels of pro-inflammatory cytokines, IL- 6 and TNF- $\alpha$, in the brain dialysate were measured by ELISA. The results indicated that the protein levels of IL-6 and TNF- $\alpha$ in the brain dialysate were markedly increased in the model group, compared with the control and sham groups $(\mathrm{P}<0.05$; Fig. 5). However, AS treatment significantly decreased the levels of IL- 6 and TNF- $\alpha$ in the brains dialysate of $A \beta$-treated rats. These results suggest that AS reduced the level of the pro-inflammatory cytokines, IL-6 and TNF- $\alpha$, in the brains of $A \beta$-treated rats.

AS treatment decreases caspase-3 and increases Bcl-2 expression in $A \beta$-treated rats. To investigate the effects of $A \beta$ oligomers and AS treatment on apoptosis-associated proteins, the expression levels of caspase- 3 and Bcl-2 in the hippocampus of rats were detected by western blot analysis. The results showed that the expression of caspase- 3 was increased and the expression of Bcl-2 was decreased in the model group, when compared with that in the control and sham groups $(\mathrm{P}<0.01$; Fig. 6). However, when treated with AS, the expression of caspases-3 was significantly decreased, and the expression of Bcl-2 was significantly increased in the AD model rats 


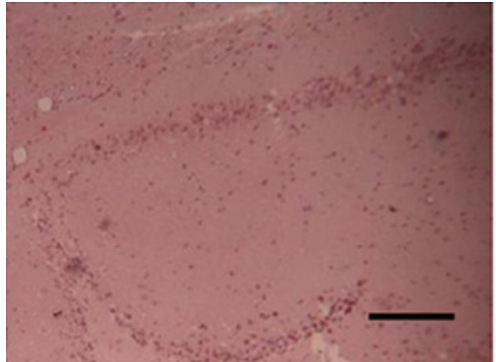

Control

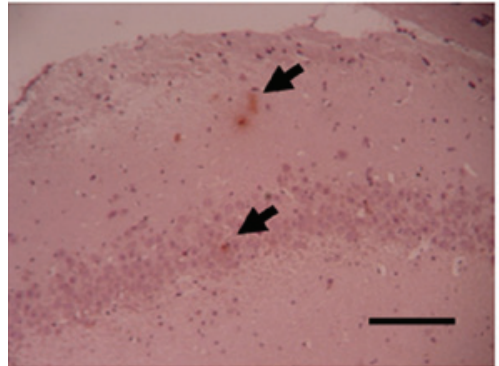

$5 \mathrm{mg} / \mathrm{kg}$ AS

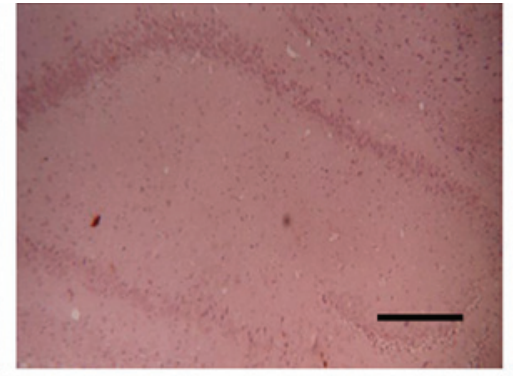

Sham

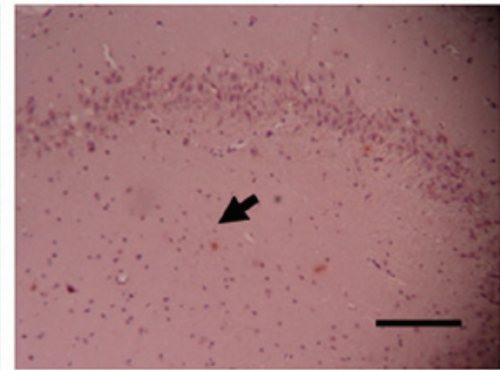

$15 \mathrm{mg} / \mathrm{kg} \mathrm{AS}$

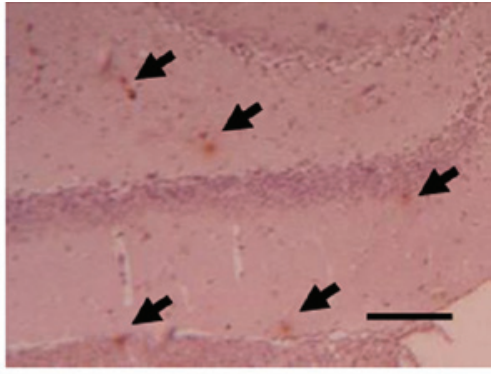

Model

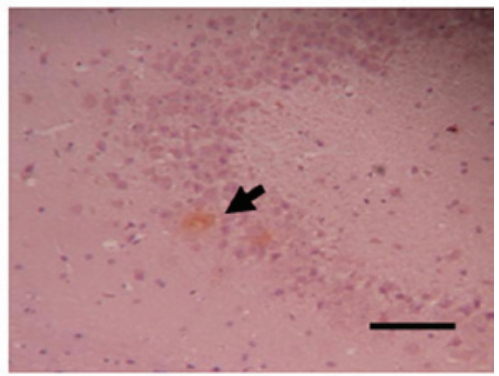

$45 \mathrm{mg} / \mathrm{kg}$ AS

Figure 3. AS treatment decreased $A \beta$ deposition in hippocampus in $A \beta$-treated rats. Immunohistochemical analysis was performed to detect the $A \beta$ deposition in hippocampus in the control, sham, model and AS treatment $(5,15$ or $45 \mathrm{mg} / \mathrm{kg}$ body weight) groups. Arrows indicated neuritic plaques. Scale bar, $10 \mu \mathrm{m}$. AS, asiaticoside; $\mathrm{A} \beta, \beta$-amyloid.

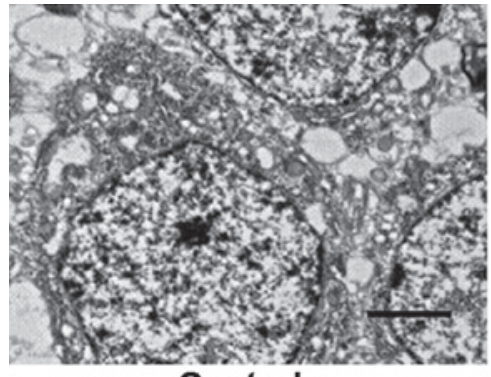

Control

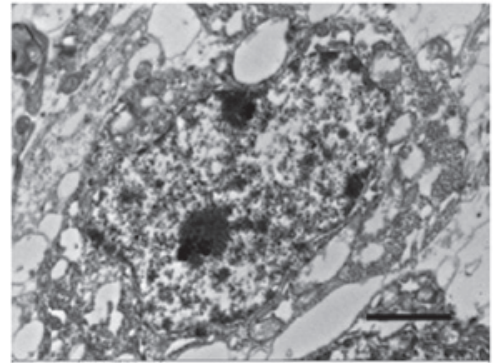

$5 \mathrm{mg} / \mathrm{kg}$ AS

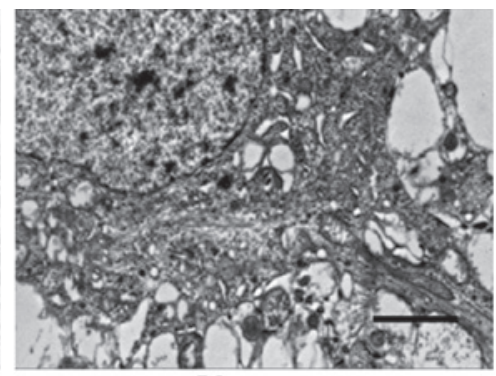

Sham

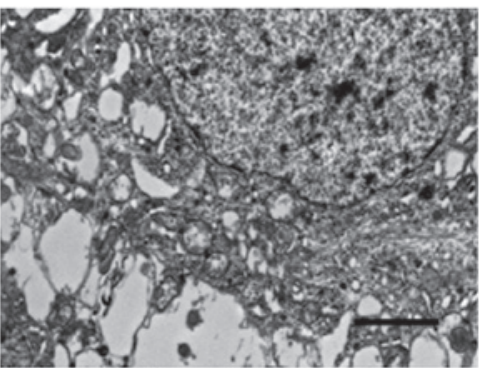

$15 \mathrm{mg} / \mathrm{kg}$ AS

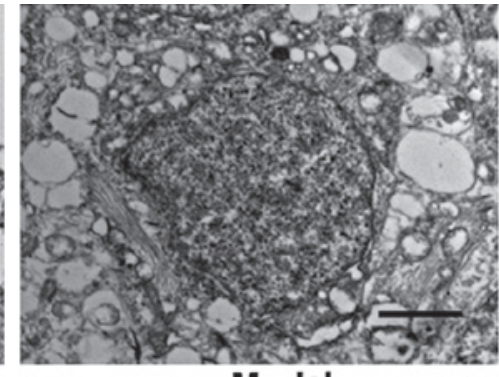

Model

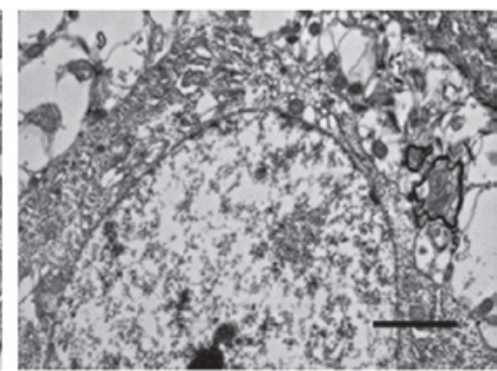

$45 \mathrm{mg} / \mathrm{kg}$ AS

Figure 4. AS treatment ameliorated the impaired subcellular structure in the brains of A $\beta$-treated rats. Transmission electron microscopy was performed to detect the A $\beta$ deposition in the hippocampus of rats in the control, sham, model and AS treatment $(5,15$ or $45 \mathrm{mg} / \mathrm{kg}$ body weight) groups. Scale bar, $2 \mu \mathrm{m}$. AS, asiaticoside; $\mathrm{A} \beta, \beta$-amyloid.

$(\mathrm{P}<0.01$; Fig. 6). These results suggest that AS treatment altered the expression levels of apoptosis-associated proteins in rats treated with $A \beta$ oligomers, which may contribute to its protective effects in the disease pathogenesis.

\section{Discussion}

$\mathrm{AD}$ is a progressive neurodegenerative disorder, clinically characterized by the impairment of cognitive function and the changes in behavior and personality $(22,23)$. One hallmark of $\mathrm{AD}$ is the amyloid deposition, the major component of which is $A \beta$ peptide with $40-42$ residues (24). The pathogenic factors for $\mathrm{AD}$ include oxidative stress, reactive oxygen species cytotoxicity, mitochondrial dysfunction and apoptosis, and inflammatory responses (25-27). Excessive accumulation of $A \beta$ oligomers in the brain serves an important role in the disease pathogenesis, particularly concerning cognitive deficits. 

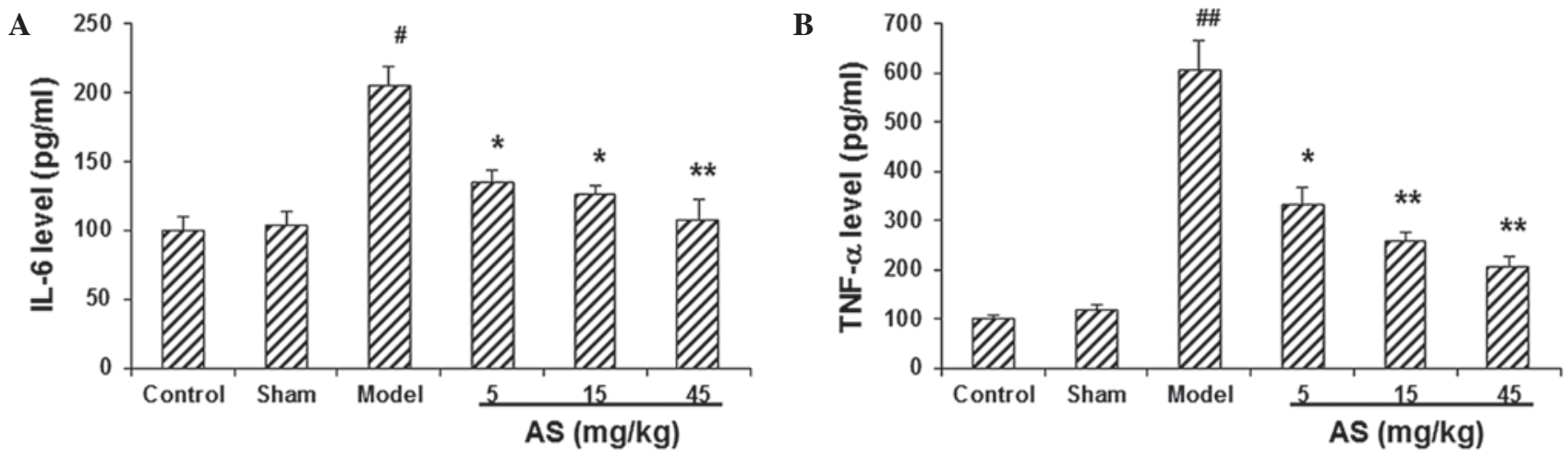

Figure 5. AS treatment reduced the levels of the pro-inflammatory cytokines (A) IL-6 and (B) TNF- $\alpha$ in the brains of A $\beta$-treated rats. The levels in the brain dialysate were measured by enzyme-linked immunosorbent assay. ${ }^{\#} \mathrm{P}<0.05$ and ${ }^{\# \#} \mathrm{P}<0.01$ vs. control group; ${ }^{*} \mathrm{P}<0.05$ and ${ }^{* *} \mathrm{P}<0.01$, vs. model group. AS, asiaticoside; IL-6, interleukin-6; TNF, tumor necrosis factor; A $\beta, \beta$-amyloid.
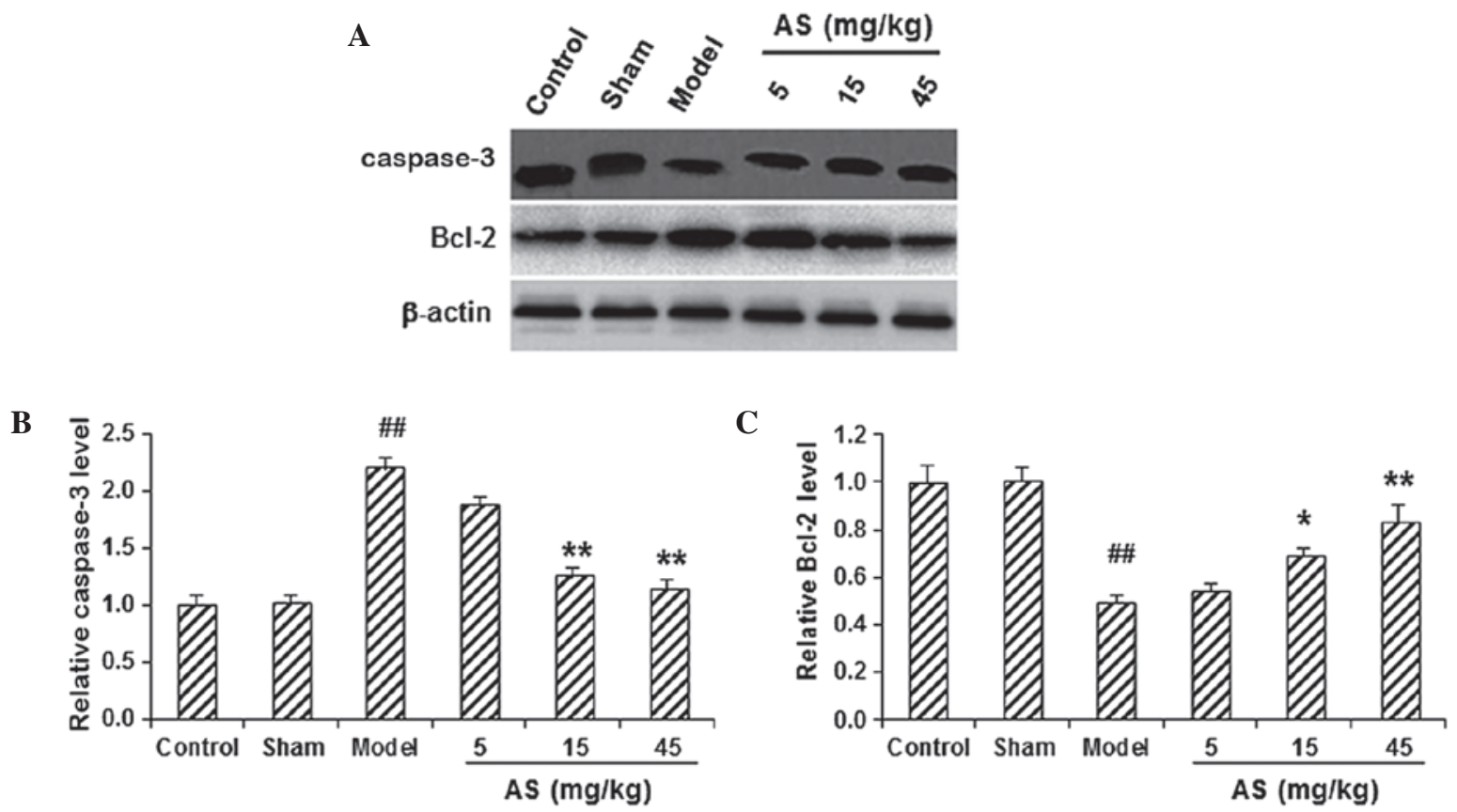

Figure 6. AS treatment decreased caspase- 3 expression and increased Bcl-2 expression in A $\beta$-treated rats. (A) The expression levels of caspase- 3 and Bcl-2 in the hippocampus were detected by western blot analysis. Statistical analysis graphs of the expression levels of (B) caspase-3 and (C) Bcl-2 are shown. ${ }^{\# \#<0.01}$ vs. control group; ${ }^{*} \mathrm{P}<0.05$ and ${ }^{* *} \mathrm{P}<0.01$, vs. model group. AS, asiaticoside; $\mathrm{Bcl}-2$, B-cell lymphoma-2; $\mathrm{A} \beta, \beta$-amyloid.

Previous studies have indicated that intracerebroventricular injection of $A \beta$ 1-42 oligomers at a nanomolar dose can dramatically impair the learning and memory function in rats (28), accompanied by increased apoptosis in the hippocampus and high levels of pro-inflammation cytokines in the brain $(29,30)$. Furthermore, intracerebroventricular injection of low doses of $\mathrm{A} \beta$ 25-35 (31) or A $\beta$ 1-28 $(32,33)$ has also been shown to influence the spatial memory function and Y-maze alternation behavior in mice. MWM spatial learning test is typically used in the evaluation of rodent models for cognitive disorders and possible treatments $(34,35)$. In the present study, $A \beta 1-42$ oligomers were administrated to establish a AD rat model, and the spatial learning and memory function of rats was assessed by the MWM test. The effects of AS administration on the disease pathogenesis and the associated mechanisms of its effect were also investigated. The results revealed that, on days 4-5, cognitive and memory functionality reduction was observed in the rats treated with $\mathrm{A} \beta$ oligomers.

Centella asiatica (L.) Urban (Umbelliferae), which is widely distributed in south China, has been used as a folk medicine agent for the improvement of learning and memory ability, and for treating neurological disorders $(1,36)$. AS is one of the active ingredients of Centella asiatica, responsible for its pharmacological activity. AS exerts various therapeutic effects, such as heat removal, diuresis promotion, detumescence and detoxification, and has been used in the treatment of urinary tract infections, skin diseases, jaundice, diarrhea, measles and larynx gall injuries (37). Although a recent study has demonstrated that AS also exerts nerve protective effects (38), its role on $\mathrm{AD}$ pathogenesis and the associate mechanisms of its action, particularly concerning the pro-inflammatory cytokine levels and subcellular structure, have not been fully elucidated (39). The results of the present study revealed that 
the escape latency time was significantly longer in the model group compared with that in the AS-treated group. In the probe test, the time the rats in the model group spent in the target quadrant and the swimming distance recorded were significantly reduced, when compared with those in the AS treatment groups. These findings suggested that AS was able to ameliorate the declined cognitive function in rats treated with $A \beta$ oligomers, and attenuated the neurotoxicity induced by the peptides.

The mitochondrial cascade hypothesis claims that mitochondrial dysfunction is the primary and leading event in AD pathology (7). Evidence shows that the progressive accumulation of $\mathrm{A} \beta$ peptides in mitochondria may induce mitochondrion-mediated toxicity (40). Along with energy metabolism, mitochondria serve a pivotal role in the survival and death of neurons through the regulation of the apoptotic pathways, by releasing cytochrome $c$ and other pro-apoptotic factors (41). In the present study, TEM analysis demonstrated that the structure of nuclei and mitochondria in the hippocampus of the control and sham groups was intact, with a complete membrane observed; by contrast, in the model group, the subcellular structure was evidently damaged. Following treatment with AS, the injuries in the mitochondria in hippocampal neurons was restored in these model rats.

To investigate the possible mechanisms underlying the protective effects of AS against AD, the levels of pro-inflammatory factors IL- 6 and TNF- $\alpha$ in the rat brains were measured with microdialysis probes. Pro-inflammatory cytokines serve important roles in AD pathogenesis, and the alterations in their serum levels have been observed in AD patients $(42,43)$. The inflammatory response associated with the activation of microglia and astrocytes is an important factor in AD pathology, as evidenced by post-mortem analysis of AD brains and studies in animal models (44-46). Inflammatory response is presumably triggered by soluble $\mathrm{A} \beta$ peptides or fibrils, leading to microglial activation, particularly in the vicinity of neuritic plaques. These activated microglia exhibit altered morphology, and produce interleukins, interferons, chemokines and components of the complement system $(47,48)$. Pro-inflammatory cytokines released by activated microglia, including IL-1 $\beta$, IL-6, IL-8 and TNF- $\alpha$, have been implicated in neurodegeneration (49). In the present study, the contents of IL- 6 and TNF- $\alpha$ in the brain dialysate were found to be markedly increased in the model group, when compared with the control and sham groups, while treatment with AS decreased the levels of these pro-inflammatory cytokines.

Numerous studies have demonstrated that $A \beta$ is a predominant factor in the pathogenesis of $\mathrm{AD}$, and that cellular apoptosis is involved in the disease pathology (50). Caspase- 3 and $\mathrm{Bcl}-2$ are important participants in the process of apoptosis, and their expression levels can determine the cell survival and death (51). Activated caspase-3 has been reported to be present in AD brains $(44,52)$ and amyloid precursor protein transgenic mice $(53,54)$. Notably, $A \beta 1-42$ has been shown to induce cytochrome $c$ release from mitochondria (55), which can activate caspase-3 and initiate apoptosis. By contrast, Bcl-2 is an apoptosis-inhibiting protein, which prevents cellular apoptosis. In the current study, the expression levels of caspase- 3 and Bcl-2 were also detected. The current study results demonstrated that AS treatment decreased the expression of caspase- 3 and increased the expression of Bcl-2 in the $\mathrm{AD}$ model rats.

In conclusion, the results of the present study revealed that AS exerted neuroprotective effects in an AD rat model induced by intracerebroventricular injection of $A \beta$ 1-42 oligomers, in a dose-dependent manner. AS alleviated the impairment in learning and memory function, decreased the $\mathrm{A} \beta$ deposition in the hippocampus and restored the damage in the subcellular structure. Furthermore, the results showed that AS reduced the pro-inflammatory factor levels of IL-6 and TNF- $\alpha$, decreased the expression levels of caspase- 3 and increased the expression levels of $\mathrm{Bcl}-2$. These results suggest that AS has a notable protective effects on $\mathrm{A} \beta$-induced AD pathology, and the mechanism underlying its action may be associated with the alleviation of the mitochondrial injuries, its anti-inflammatory activities and its effect on the expression levels of apoptosis-associated proteins.

\section{Acknowledgements}

The presents study was supported by funding from the Science and Technology Department of Sichuan Province (grant no. 2014SZ0071) and the Educational Commission of Sichuan Province (grant nos. 14ZA0143 and 13ZB0267).

\section{References}

1. Qi FY, Yang L, Tian Z, Zhao MG, Liu SB and An JZ: Neuroprotective effects of Asiaticoside. Neural Regen Res 9: 1275-1282, 2014.

2. Zhang Zhuo, Liu Minghua, Zhang Hong, Wu Jianbo and Wan Jinyuan: Effects of Aisaticoside on Apoptosis of PC12 cells Induced by $\beta$-amylnid Peptide. Chinese Journal of New Drugs 21: 206-210, 2012.

3. Zhang L, Li HZ, Gong X, Luo FL, Wang B, Hu N, Wang CD, Zhang $Z$ and Wan JY: Protective effects of Asiaticoside on acute liver injury induced by lipopolysaccharide/D-galactosamine in mice. Phytomedicine 17: 811-819, 2010.

4. Miklossy J, Kis A, Radenovic A, Miller L, Forro L, Martins R, Reiss K, Darbinian N, Darekar P, Mihaly L and Khalili K: Beta-amyloid deposition and Alzheimer's type changes induced by Borrelia spirochetes. Neurobiol Aging 27: 228-236, 2006.

5. Tomic JL, Pensalfini A, Head E and Glabe CG: Soluble fibrillar oligomer levels are elevated in Alzheimer's disease brain and correlate with cognitive dysfunction. Neurobiol Dis 35: 352-358, 2009

6. Hroudová J, Singh N and Fišar Z: Mitochondrial dysfunctions in neurodegenerative diseases: Relevance to Alzheimer's disease. Biomed Res Int 2014: 175062, 2014.

7. Picone P, Nuzzo D, Caruana L, Scafidi V and Di Carlo M: Mitochondrial dysfunction: Different routes to Alzheimer's disease therapy. Oxid Med Cell Longev 2014: 780179, 2014.

8. Ridge PG, Koop A, Maxwell TJ, Bailey MH, Swerdlow RH, Kauwe JS and Honea RA; Alzheimer's Disease Neuroimaging Initiative: Mitochondrial haplotypes associated with biomarkers for Alzheimer's disease. PLoS One 8: e74158, 2013.

9. Moreira PI, Carvalho C, Zhu X, Smith MA and Perry G: Mitochondrial dysfunction is a trigger of Alzheimer's disease pathophysiology. Biochim Biophys Acta 1802: 2-10, 2010.

10. Lin MT and Beal MF: Mitochondrial dysfunction and oxidative stress in neurodegenerative diseases. Nature 443: 787-795, 2006.

11. Sas K, Robotka H, Toldi J and Vécsei L: Mitochondria, metabolic disturbances, oxidative stress and the kynurenine system, with focus on neurodegenerative disorders. J Neurol Sci 257: 221-239, 2007.

12. Frautschy SA, Hu W, Kim P, Miller SA, Chu T, Harris-White ME and Cole GM: Phenolic anti-inflammatory antioxidant reversal of Abeta-induced cognitive deficits and neuropathology. Neurobiol Aging 22: 993-1005, 2001. 
13. Hu S, Ying Z, Gomez-Pinilla F and Frautschy SA: Exercise can increase small heat shock proteins (sHSP) and pre- and post-synaptic proteins in the hippocampus. Brain Res 1249: 191-201, 2009.

14. Srivareerat M, Tran TT, Salim S, Aleisa AM and Alkadhi KA: Chronic nicotine restores normal $A \beta$ levels and prevents short-term memory and E-LTP impairment in A $\beta$ rat model of Alzheimer's disease. Neurobiol Aging 32: 834-844, 2011.

15. Srivareerat M, Tran TT, Alzoubi KH and Alkadhi KA: Chronic psychosocial stress exacerbates impairment of cognition and long-term potentiation in beta-amyloid rat model of Alzheimer's disease. Biol Psychiatry 65: 918-926, 2009.

16. Yan JJ, Cho JY, Kim HS, Kim KL, Jung JS, Huh SO, Suh HW, Kim YH and Song DK: Protection against beta-amyloid peptide toxicity in vivo with long-term administration of ferulic acid. $\mathrm{Br}$ J Pharmacol 133: 89-96, 2001.

17. Loane DJ, Pocivavsek A, Moussa CE, Thompson R, Matsuoka Y, Faden AI, Rebeck GW and Burns MP: Amyloid precursor protein secretases as therapeutic targets for traumatic brain injury. Nat Med 15: 377-379, 2009.

18. Shang JL, Cheng Q, Yang WF, Zhang M, Cui Y and Wang YF Possible roles of COX-1 in learning and memory impairment induced by traumatic brain injury in mice. Braz J Med Biol Res 47: 1050-1056, 2014.

19. Li J, Wang C, Zhang JH, Cai JM, Cao YP and Sun XJ: Hydrogen-rich saline improves memory function in a rat model of amyloid-beta-induced Alzheimer's disease by reduction of oxidative stress. Brain Res 1328: 152-161, 2010.

20. Saarinen NM, Abrahamsson A and Dabrosin C: Estrogen-induced angiogenic factors derived from stromal and cancer cells are differently regulated by enterolactone and genistein in human breast cancer in vivo. Int J Cancer 127: 737-745, 2010.

21. Xu BJ, Yan W, Jovanovic B, Shaw AK, An QA, Eng J, Chytil A, Link AJ and Moses HL: Microdialysis combined with proteomics for protein identification in breast tumor microenvironment in vivo. Cancer Microenviron 4: 61-71, 2010

22. Cleary JP, Walsh DM, Hofmeister JJ, Shankar GM Kuskowski MA, Selkoe DJ and Ashe KH: Natural oligomers of the amyloid-beta protein specifically disrupt cognitive function Nat Neurosci 8: 79-84, 2005

23. Shah RS, Lee HG, Xiongwei Z, Perry G, Smith MA and Castellani RJ: Current approaches in the treatment of Alzheimer's disease. Biomed Pharmacother 62: 199-207, 2008.

24. Boekhoorn K, Joels M and Lucassen PJ: Increased proliferation reflects glial and vascular-associated changes, but not neurogenesis in the presenile Alzheimer hippocampus. Neurobiol Dis 24: 1-14, 2006

25. Weiner HL and Selkoe DJ: Inflammation and therapeutic vaccination in CNS diseases. Nature 420: 879-884, 2002.

26. Wang J, Zhang HY and Tang XC: Cholinergic deficiency involved in vascular dementia: Possible mechanism and strategy of treatment. Acta Pharmacol Sin 30: 879-888, 2009.

27. Bennett S, Grant MM and Aldred S: Oxidative stress in vascular dementia and Alzheimer's disease: A common pathology. J Alzheimers Dis 17: 245-257, 2009.

28. Li J, Wang C, Zhang JH, Cai JM, Cao YP and Sun XJ: Hydrogen-rich saline improves memory function in a rat model of amyloid-beta-induced Alzheimer's disease by reduction of oxidative stress. Brain Res 1328: 152-161, 2010.

29. Liu T, Huang Y, Chen J, Chi H, Yu Z, Wang J and Chen C: Attenuated ability of BACE1 to cleave the amyloid precursor protein via silencing long noncoding RNA BACE1-AS expression. Mol Med Rep 10: 1275-1281, 2014.

30. Kuo YC and Liu YC: Cardiolipin-incorporated liposomes with surface CRM197 for enhancing neuronal survival against neurotoxicity: Int J Pharm 473: 334-344, 2014.

31. Shen YX, Xu SY, Wei W, Wang XL, Wang H and Sun X: Melatonin blocks rat hippocampal neuronal apoptosis induced by amyloid beta-peptide 25-35. J Pineal Res 32: 163-167, 2002.

32. Baruch-Suchodolsky R and Fischer B: Soluble amyloid beta1-28-copper(I)/copper(II)/Iron(II) complexes are potent antioxidants in cell-free systems. Biochemistry 47: 7796-7806, 2008

33. Cribbs DH, Velazquez P, Soreghan B, Glabe CG and Tenner AJ: Complement activation by cross-linked truncated and chimeric full-length beta-amyloid. Neuroreport 8: 3457-3462, 1997.
34. Alipanahzadeh H, Soleimani M, Soleimani Asl S, Pourheydar B, Nikkhah A and Mehdizadeh M: Transforming growth factor- $\alpha$ improves memory impairment and neurogenesis following ischemia reperfusion. Cell J 16: 315-324, 2014.

35. Choi JH, Park P, Baek GC, Sim SE, Kang SJ, Lee Y, Ahn SH, Lim CS, Lee YS, Collingridge GL and Kaang BK: Effects of $\mathrm{PI} 3 \mathrm{~K} \gamma$ overexpression in the hippocampus on synaptic plasticity and spatial learning. Mol Brain 7: 78, 2014.

36. Xu CL, Wang QZ, Sun LM, Li XM, Deng JM, Li LF, Zhang J, $\mathrm{Xu}$ R and Ma SP: Asiaticoside: Attenuation of neurotoxicity induced by MPTP in a rat model of Parkinsonism via maintaining redox balance and up-regulating the ratio of $\mathrm{Bcl}-2 / \mathrm{Bax}$. Pharmacol Biochem Behav 100: 413-418, 2012.

37. Somboonwong J, Kankaisre M, Tantisira B and Tantisira MH: Wound healing activities of different extracts of Centella asiatica in incision and burn wound models: An experimental animal study. BMC Complement Altern Med 12: 103, 2012.

38. Mook-Jung I, Shin JE, Yun SH, Huh K, Koh JY, Park HK, Jew SS and Jung MW: Protective effects of asiaticoside derivatives against beta-amyloid neurotoxicity. J Neurosci Res 58: 417-425, 1999.

39. Wang YT, Lu XM, Chen KT, Shu YH and Qiu CH: Immunotherapy strategies for spinal cord injury. Curr Pharm Biotechnol 16: 492-505, 2015

40. Walsh DM and Selkoe DJ: A beta oligomers-a decade of discovery. J Neurochem 101: 1172-1184, 2007.

41. Chen JX and Yan SS: Role of mitochondrial amyloid-beta in Alzheimers disease. J Alzheimers Dis 20 (Suppl 2): S569-S578, 2010.

42. François A, Terro F, Janet T, Rioux Bilan A, Paccalin M and Page G: Involvement of interleukin- $1 \beta$ in the autophagic process of microglia: Relevance to Alzheimer's disease. J Neuroinflammation 10: 151, 2013.

43. Khemka VK, Ganguly A, Bagchi D, Ghosh A, Bir A, Biswas A, Chattopadhyay $S$ and Chakrabarti S: Raised serum proinflammatory cytokines in Alzheimer's disease with depression. Aging Dis 5: 170-176, 2014.

44. Lin LX, Bo XY, Tan YZ, Sun FX, Song M, Zhao J, Ma ZH, Li M, Zheng KJ and Xu SM: Feasibility of $\beta$-sheet breaker peptide-H102 treatment for Alzheimer's disease based on $\beta$-amyloid hypothesis. PLoS One 9: e112052, 2014

45. Trepanier $\mathrm{CH}$ and Milgram NW: Neuroinflammation in Alzheimer's disease: Are NSAIDs and selective COX-2 inhibitors the next line of therapy? J Alzheimers Dis 21: 1089-1099, 2010.

46. Pan J, Michalec M and Louis ED: Non-Steroidal anti-inflammatory drug use and essential tremor. Neuroepidemiology 43: 145-149, 2014.

47. Weisman D, Hakimian E and Ho GJ: Interleukins, inflammation, and mechanisms of Alzheimer's disease. Vitam Horm 74: 505-530, 2006

48. Floden AM and Combs CK: Beta-amyloid stimulates murine postnatal and adult microglia cultures in a unique manner. $\mathrm{J}$ Neurosci 26: 4644-4648, 2006.

49. Murray CL, Skelly DT and Cunningham C: Exacerbation of CNS inflammation and neurodegeneration by systemic LPS treatment is independent of circulating IL-1 $\beta$ and IL-6. J Neuroinflammation 8: 50, 2011

50. Du H, Shi D and Yan S: Unlocking the Door to Neuronal Woes in Alzheimer's Disease: $\mathrm{A} \beta$ and Mitochondrial Permeability Transition Pore. Pharmaceuticals (Basel), 3: 1936-1948, 2010.

51. Casal C, Serratosa J and Tusell JM: Effects of beta-AP peptides on activation of the transcription factor NF-kappaB and in cell proliferation in glial cell cultures. Neurosci Res 48: 315-323, 2004.

52. Reynolds CH, Nebreda AR, Gibb GM, Utton MA and Anderton BH: Reactivating kinase/p38 phosphorylates tau protein in vitro. J Neurochem 69: 191-198, 1997.

53. Zhang Y, Pan C, Wu X, Dong Y, Culley DJ, Crosby G, Li T and Xie Z: Different effects of anesthetic isoflurane on caspase-3 activation and cytosol cytochrome $\mathrm{c}$ levels between mice neural progenitor cells and neurons. Front Cell Neurosci 8: 14, 2014.

54. Zhang QL, Jia L, Jiao X, Guo WL, Ji JW, Yang HL and Niu Q: APP/PS1 transgenic mice treated with aluminum: An update of Alzheimer's disease model. Int J Immunopathol Pharmacol 25: 49-58, 2012.

55. Ott M, Robertson JD, Gogvadze V,Zhivotovsky B and Orrenius S: Cytochrome c release from mitochondria proceeds by a two-step process. Proc Natl Acad Sci USA 99: 1259-1263, 2002. 